\title{
ESTUDO PRELIMINAR EM CFD PARA COMPARAÇÃO DA ALIMENTAÇÃO DE UM TRANSPORTADOR PENEUMÁTICO COM DISPOSITIVO VENTURI EM DIFERENTES ORIENTAÇÕES
}

\author{
T. F. PÁDUA*, R. BÉTTEGA, J. T. FREIRE \\ Universidade Federal de São Carlos, Departamento de Engenharia Química \\ *e-mail: tfpadua@gmail.com
}

\begin{abstract}
RESUMO
A secagem e o pré-aquecimento de material sólido podem ser realizados em transportador pneumático. A alimentação de material sólido diretamente em trecho vertical reduz a abrasão e perda de carga no transportador, podendo representar uma vantagem frente à abordagem comumente encontrada, que envolve a inserção de material em trecho horizontal e posteriormente introduz a corrente gás-sólido em trecho vertical através de uma curva. Entretanto, as distribuições axial e radial do sólido para operações desse tipo e com partículas grosseiras foram pouco estudadas e não há discussões no que tange à homogeneidade promovida pelas distintas orientações do dispositivo na região próxima e distante da alimentação. $\mathrm{O}$ objetivo deste trabalho foi o de avaliar o escoamento multifásico com alimentação utilizando dispositivo Venturi em trecho vertical e horizontal através de simulações em CFD com modelo Euler-Euler. As simulações indicaram heterogeneidade no sistema para a região próxima à alimentação. A alimentação na vertical apresentou-se como aquela a fornecer maior homogeneidade na fase sólida para o trecho ascendente, sendo a mais indicada segundo as simulações.
\end{abstract}

\section{INTRODUÇÃO}

O transporte pneumático é uma alternativa para secagem e preaquecimento de material sólido (Rajan et al., 2008; Hidayat et al, 2007). A secagem de cereais e fármacos está entre os tipos de operação que podem se beneficiar com o desenvolvimento do processo, que na literatura é também chamada secagem flash.

As vantagens da secagem em transportador pneumático incluem o fato de $\mathrm{o}$ material ser transportado enquanto a secagem e o aquecimento ocorrem, além da grande área de contato gás-sólido.

Muitas das aplicações no aquecimento e secagem incluem partículas grosseiras (maiores do que $0,5 \mathrm{~mm}$ de diâmetro). Além disso, existem aplicações da secagem de pastas, que envolvem o recobrimento de material particulado grosseiro (Souza et al., 2010). Entretanto, a maioria dos estudos sobre o transporte pneumático em trecho ascendente foca principalmente condições para aplicações tradicionais da configuração em leito fluidizado circulante, cuja principal diferença está na faixa de diâmetros do material particulado, que neste caso varia usualmente de 60 a $80 \mu \mathrm{m}$ (Du et al., 2004; Grace \& Taghipour, 2004). Deste modo, detalhes com relação à distribuição axial e radial de sólidos em transportadores com configurações comuns à secagem são pouco conhecidos.

Muitas configurações de transportador e alimentadores para particulados grosseiros têm sido propostas. Uma configuração 
comum é a utilização de alimentação em trecho horizontal, seguida de uma curva para alimentação da mistura gás-sólido em trecho vertical, em que geralmente se utiliza um dispositivo de alimentação com afunilamento (Venturi) para evitar o desvio da corrente principal de transporte pelo conduto de alimentação (Rajan et al., 2008; Rajan et al., 2010).

Alternativamente, a alimentação diretamente em trecho vertical é uma configuração interessante. Neste caso, uma curva é evitada, reduzindo a abrasão e a perda de carga no sistema.

Lopes et al. (2011) utilizou um dispositivo Venturi em orientação vertical para a alimentação de sólidos. Além disso, dentre estas configurações está o alimentador tipo jorro (Souza et al., 2010; Costa et al, 2004; Grbavcic et al., 2006).

As maiores taxas de transporte de calor e massa ocorrem na região de aceleração, onde a velocidade de deslizamento (slip velocity) é elevada (Rajan et al., 2006). Deste modo, é importante conhecer a fluidodinâmica próxima à alimentação. Ademais, várias questões relativas à homogeneidade e mistura nos diversos sistemas para secagem em transportador pneumático continuam em aberto. Assim, uma discussão interessante está relacionada à comparação entre sistemas horizontais e verticais de alimentação.

Este trabalho teve o objetivo de avaliar o comportamento da fase sólida através de simulações Eulerianas de variáveis relacionadas à hidrodinâmica do alimentador Venturi e do transportador.

Os resultados das simulações com alimentação na vertical foram enviados para publicação em periódico (Pádua et al. 2015), onde mais detalhes podem ser obtidos. Porém, o objetivo do presente trabalho é a comparação do comportamento da fase sólida com alimentação na horizontal seguida de uma curva com a alimentação diretamente em trecho vertical. Ambas as simulações foram realizadas utilizando alimentação com dispositivo Venturi.

\section{MÉTODOS NUMÉRICO E MODELOS}

\subsection{Condições experimentais e sistema simulado.}

A Figura 1 apresenta o sistema a ser simulado. As simulações tiveram como foco o dispositivo de alimentação e o trecho ascendente.

Figura 1 - Transportador pneumático circulante utilizado.

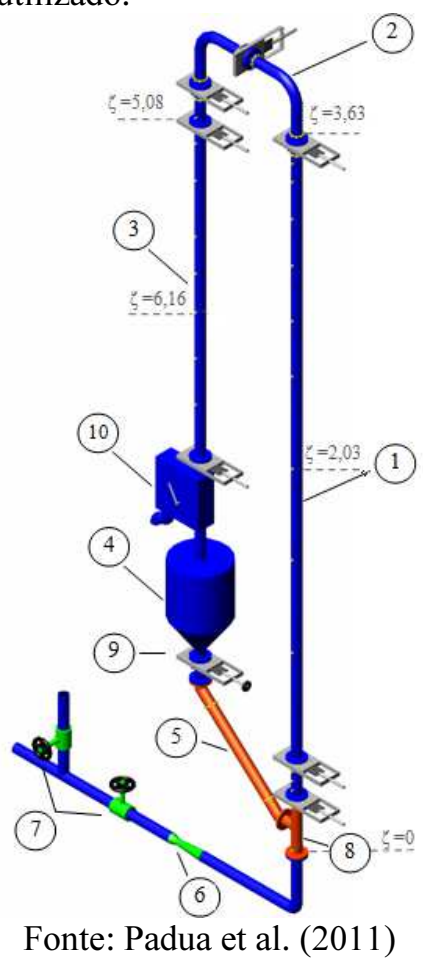

A Figura 2 apresenta as duas diferentes geometria de alimentação simuladas. O alimentador da Figura 2.a opera na vertical, enquanto o alimentador da Figura 2.b é utilizado na horizontal (trecho horizontal).

Resultados experimentais sobre a alimentação utilizando o dispositivo da Figura 2.a podem ser verificados em Pádua et 
al. 2015. No caso das simulações, foi considerada uma condição de operação intermediária à menor vazão utilizada e a máxima vazão no sistema, correspondendo à vazão de ar de $253,2 \mathrm{~m}^{3} / \mathrm{h}$ (velocidade superficial e nominal de $29,5 \mathrm{~m} / \mathrm{s}$ ) ao qual correspondem 29,6 Kg/h de sólidos transportados.

O dispositivo da Figura 2.b foi utilizado por Lopes (2011). Neste caso, a saída do reservatório era diretamente conectada à entrada do dispositivo venturi por um trecho vertical. Porém, não foi obtida condição de operação estável em transporte diluído. A ausência do transporte diluído devia-se à inviabilidade de utilizar somente uma válvula guilhotina para ajuste da vazão de sólidos, sendo necessário um dispositivo diferente para manipulação da vazão.

Figura 2 - Alimentadores simulados (cotas em $\mathrm{mm}$ ).

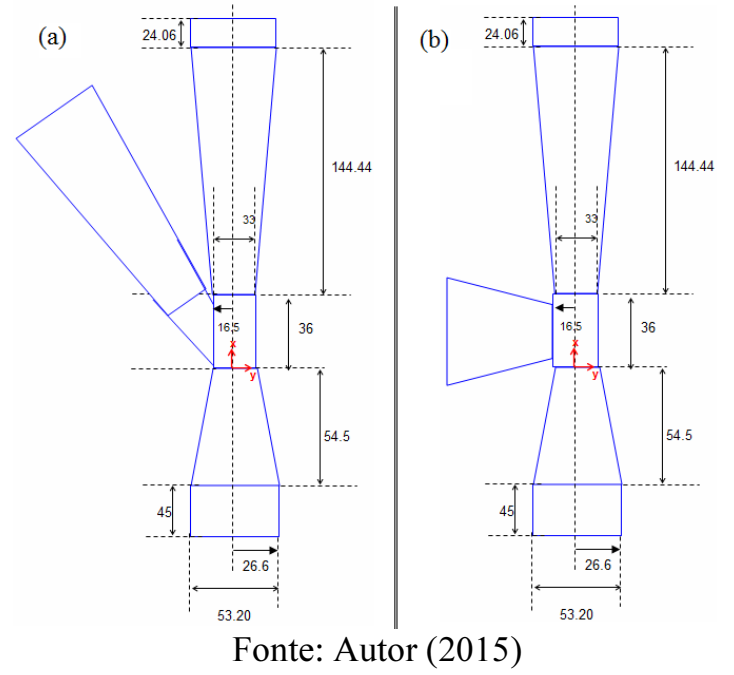

Para fins de comparação, o presente trabalho utilizará as mesmas condições experimentais para ambos os alimentadores. Assim, a condição de operação obtida para o alimentador vertical será também empreendida na simulação do alimentador horizontal, supondo que o sistema de ajuste de vazão de sólidos fosse capaz de manter tais condições de vazão, nem que fosse necessário o acoplamento à um sistema mecânico (como rosca sem fim) ou de vibração.

\subsection{Geometria e malha.}

A malha utilizada no sistema com alimentação horizontal está apresentada na Figura 3. A região de malha tetraédrica é a única exceção à malha hexaédrica no sistema e foi denominada de região de junção dos condutos. A mesma estrutura de construção foi utilizada para a alimentação na vertical e pode ser visualizada na Figura 4.

Figura 3 - Malha próxima a região de alimentação para alimentador na horizontal.

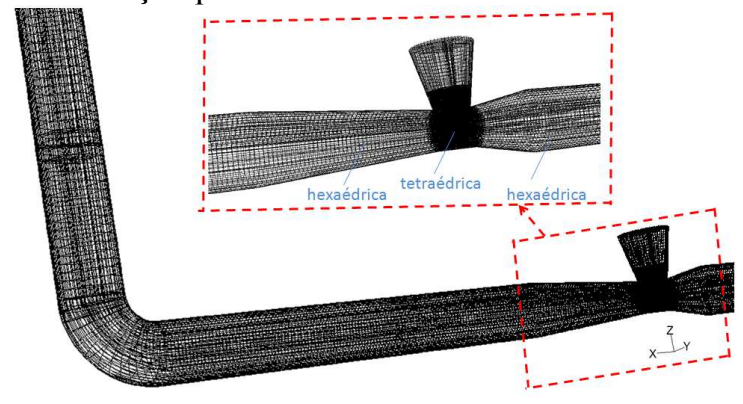

Fonte: Autor (2015)

Figura 4 - Malha próxima à alimentação para alimentador na vertical.

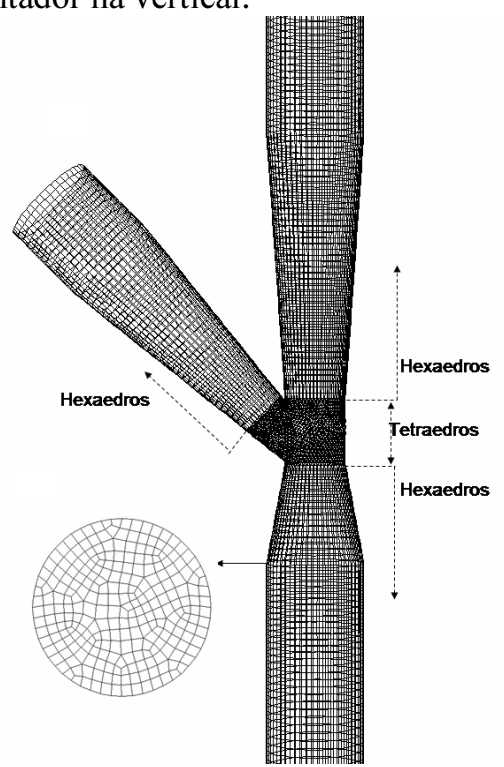

Fonte: Autor (2015). 
No caso de malhas com esta topologia, vale ressaltar que a expansão volumétrica nos troncos de cone do dispositivo Venturi garante que a malha mais refinada seja aquela presente na garganta. Assim, o refinamento da malha nessa região é representativo quanto à malha no resto do sistema.

Foram utilizadas 162506 células na simulação com alimentação na horizontal, que correspondiam à uma distância média de $2 \mathrm{~mm}$ entre nós na região de junção dos condutos. Testes de variação de malha mostraram que a resposta do escoamento (em pressão e campos de velocidade) é estável em relação às alterações próximas à região de alimentação. Mais detalhes sobre os testes de malha para o sistema na vertical podem ser obtidos em Pádua et al., 2015.

\subsection{Softwares e Métodos numéricos.}

As simulações foram realizadas em programa comercial Fluent 6.3 e geração de malha em Gambit 2.3. O Fluent 14.5, foi utilizado nas simulações com alimentador em trecho horizontal.

O modelo utilizado para o escoamento multifásico foi o de dois fluídos com modelo granular (teoria cinética) para fase particulada e adotou-se solução transiente com intervalo de 0,0001 segundos. $O$ critério de convergência para cada instante de tempo foi o resíduo escalonado máximo de $1 \times 10^{-3} \mathrm{em}$ todas as equações do modelo. Na prática, os maiores resíduos concentravam-se nas equações de momento da fase particulada, mas geralmente atingiam no máximo $2 \times 10^{-4} \mathrm{a}$ $5 \times 10^{-4}$.

Os contornos utilizados eram de velocidade prescrita para as duas entradas. $\mathrm{Na}$ entrada de ar a fração de fase granular foi considerada nula. $\mathrm{Na}$ entrada de sólidos a fração de sólidos utilizada foi de 0,6 (comum em leitos deslizantes).
A dispersão da fase sólida, estando em uma razão de carga mássica de sólidos reduzida, justifica boa parte das hipóteses dos modelos utilizados para o fechamento do sistema de equações diferenciais contínuas. Assim, assumiu-se a rápida diluição da fase sólida na alimentação.

Deste modo, o modelo de turbulência k$\varepsilon$ para fase secundária (sólidos) dispersa foi utilizado e o arraste entre as fases foi obtido por Wen \& Yu (1966). Em termos numéricos, as aproximações utilizadas na equação de momento foram de segunda ordem e para fração de fase de primeira ordem.

As soluções foram obtidas pelo método coupled SIMPLE. Além disso, utilizou-se discretização Green-Gauss baseada nos nós e demais modelos para complementação do modelo de dois fluídos incluíram: viscosidade granular (Gidaspow et al., 1992), viscosidade bulk (Lun et al., 1984).

As simulações utilizaram a formulação algébrica para temperatura granular. As simulações apresentadas utilizaram coeficiente de especularidade nulo nas paredes.

Não existem estudos relacionados ao início do processo de alimentação de sólidos no sistema. Deste modo, não há expectativa de que o início da simulação necessariamente represente o transiente real do processo. Porém, observou-se que o comportamento geral da fluidodinâmica no sistema estabilizava-se a partir de quatro segundos de escoamento. Todos os resultados são apresentados para oito segundos de escoamento simulado.

\section{RESULTADO E DICUSSÕES}

A Figura 5 apresenta os campos escalares para a fração volumétrica de sólidos em diferentes cortes do sistema com alimentação vertical em dez segundos de simulação. Os campos possuem baixa 


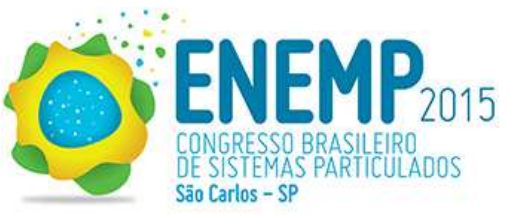

resolução devido à grande diluição dos sólidos no sistema.

Figura 5 - Campos escalares de fração de sólidos em cortes do domínio computacional para alimentação na vertical. a - corte no plano yz. $\mathrm{b}$ - corte no plano xz.
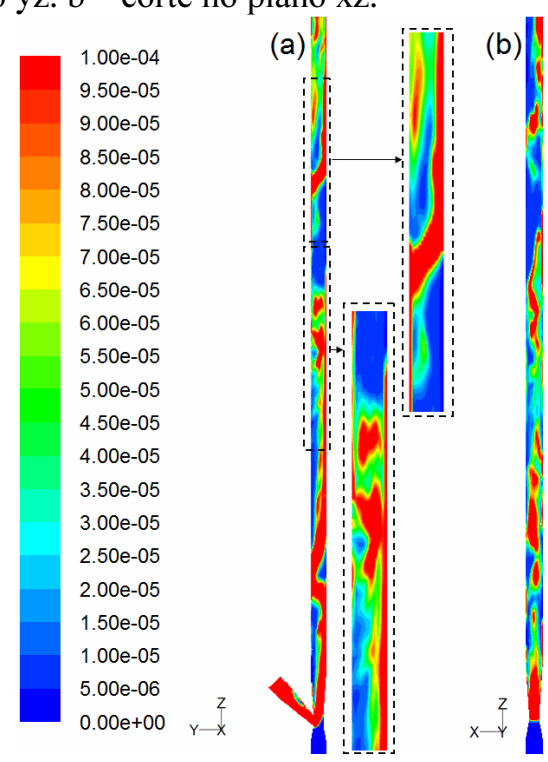

A Figura 5 indica a ocorrência de regiões de alta concentração de sólidos (agrupamentos) ao longo do trecho vertical. Naturalmente, estes agrupamentos têm concentrações muito inferiores ao observado em estudos para o regime denso nos casos de particulado fino. Ainda assim, as concentrações são elevadas o suficiente para se destacarem das proximidades mais diluídas.

A Figura 6 exemplifica a formação de alguns desses agrupamentos.

Os agrupamentos observados na Figura 5 se originavam na alimentação de sólidos de modo similar ao apresentado na Figura 6.

Deste modo, as perturbações mais próximas à inserção de material particulado geraram perturbações em secções posteriores. Assim, a fração de sólidos na região de baixa densidade próxima à alimentação é ampliada o que eleva em seguida ao aumento da fração de sólidos em regiões imediatamente posteriores ao alimentador. Em outras palavras, o aumento da concentração dos sólidos próximo à alimentação se propagava adiante no sistema.

Figura 6 - Campo escalar de fração volumétrica para alimentação na vertical. Instantes de tempo contados a partir de 9 segundos.

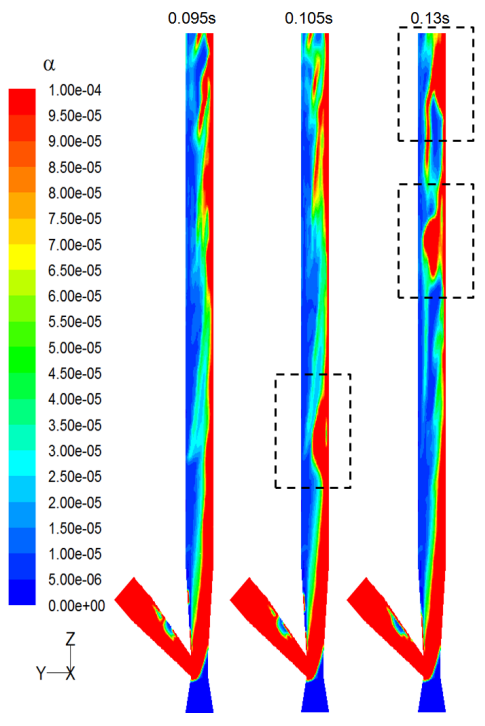

O resultado do escoamento na alimentação parece ser a eventual formação de agrupamentos de sólidos que se deslocam em relação à região densa para o centro da tubulação. Estes pequenos agrupamentos se unem às correntes densas de sólidos formando agrupamentos maiores e em região posterior.

A Figura 7 apresenta os campos escalares para a fração volumétrica de sólidos para o sistema com alimentação na orientação horizontal seguida de uma curva. É possível observar também alguns agrupamentos na entrada e saída da curva. O mesmo campo é apresentado na Figura 8, porém com frações volumétricas de sólido que chegavam a até $10^{-4}$. 


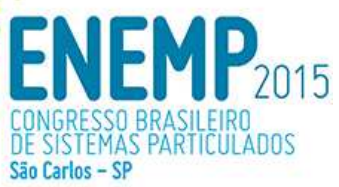

Figura 7 - Campo escalar de fração volumétrica para alimentação na horizontal. Instantes de tempo contados a partir de 9 segundos. Fração máxima de $10^{-5}$.

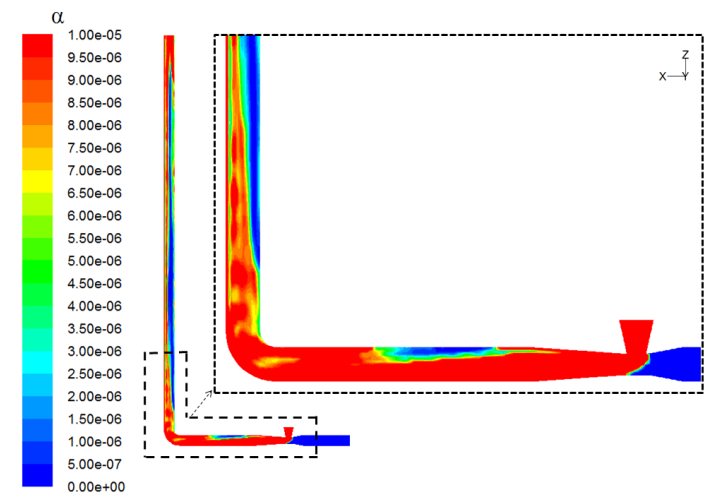

Figura 8 - Campo escalar de fração volumétrica para alimentação na horizontal. Instantes de tempo contados a partir de 9 segundos. Fração máxima de $10^{-4}$.

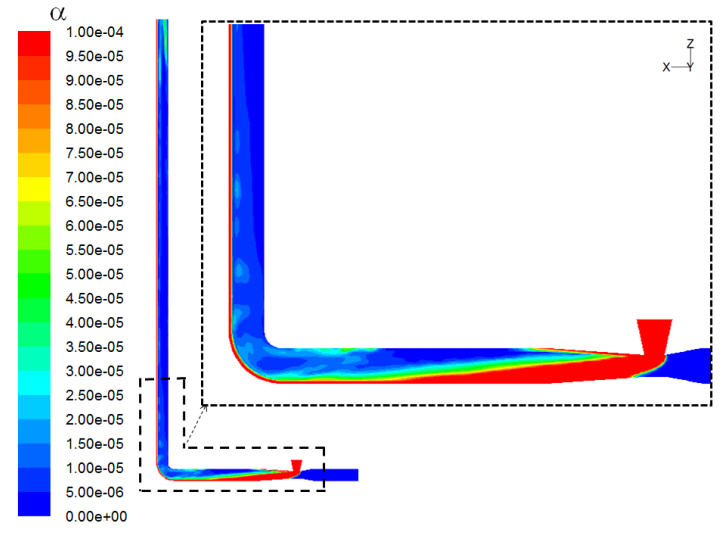

A ação gravitacional promove acumulo de material particulado na parte inferior do transportador. A alimentação na vertical também promove acumulo na região oposta à alimentação, nesse caso, exclusivamente devido ao efeito inercial da fase. Assim, em todo o caso, a região de desvio dos sólidos pelo ar no sistema ocorria do mesmo lado do conduto lateral.

A partir das figuras apresentadas, observamos que a fração de fase indica acumulo na região inferior do trecho horizontal também próximo a curva. Além disso, maiores concentrações de sólido foram visualizadas na parede externa da curva. As maiores concentrações nessa região tendem à se manter até distâncias consideráveis no trecho vertical, ou seja, não há grande homogeneização da fase sólida.

O comportamento da fase sólida em permanecer mais concentrada na região externa da curva para o trecho ascendente pode ser observada na Figura 9, que apresenta a média no tempo das frações de fase em função da posição no trecho ascendente para ambos os alimentadores.

A simulação da alimentação em trecho vertical apresentou concentrações mais uniformes no trecho ascendente, em razão da ação da curva e da inércia da fase particulada manterem altas concentrações na parede em direção externa da curva para caso da alimentação horizontal.

Apesar das análises indicarem que a alimentação vertical apresenta melhores condições de homogeneidade para operação em trecho ascendente, os resultados apresentados devem ser vistos como sendo preliminares. A literatura já indicou que modelos Euler-Euler possuem limitações quanto ao tratamento dado à fase sólida nos contornos de parede (Pirker et al., 2010), que afeta particularmente sistemas que apresentam curvas, como a alimentação na horizontal. A questão torna-se difícil na ausência de resultados experimentais detalhados quanto ao escoamento de sólidos nas condições utilizadas no presente trabalho, tanto para partículas grosseiras, como para alimentação próxima ao trecho vertical. 
Figura 9 - Fração média (temporal) de sólidos para trechos verticais utilizando dois alimentadores distintos. a - Alimentação na vertical (plano observado na Figura 5.a). b alimentação na horizontal (plano observado na Figura 8).

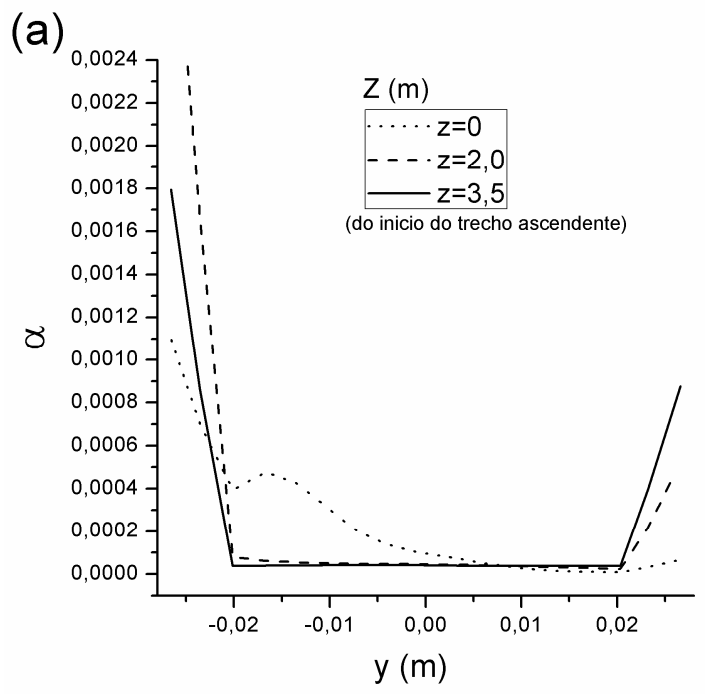

(b)

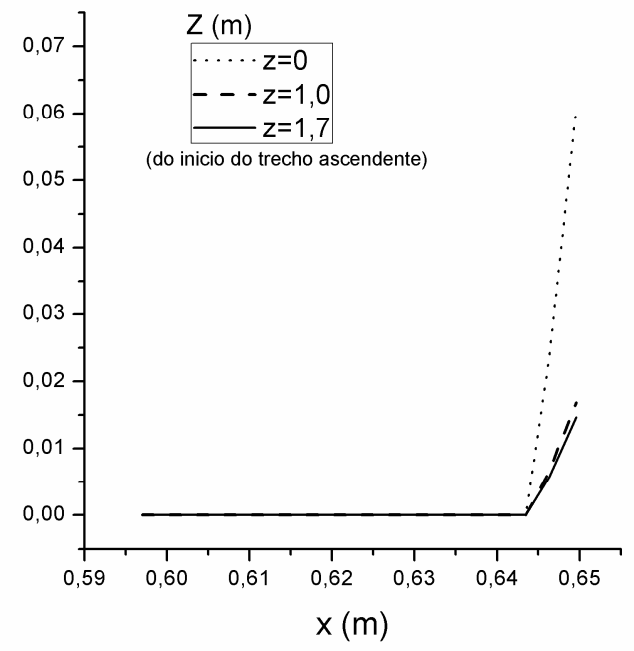

Pirker et al. (2010) obteve previsão de material particulado acumulado na região da parede por trechos longos de uma curva ao utilizar simulações Eulerianas e mostrou resultados experimentais que contradiziam suas próprias previsões. No entanto, obteve previsões mais apropriadas ao utilizar modelos híbridos. Vashisth \& Grace (2012) utilizaram uma abordagem de CFD-DEM para simular com sucessos experimentos de material particulado grosseiro em escoamento por uma curva. O principal fator para a dispersão do material sólido nesse caso era o modelo estocástico utilizado para acoplamento da turbulência.

Deste modo, como passos importantes para avaliação da questão, colocam-se como pontos chave o aprimoramento do modelo utilizado nas simulações na direção de considerar os efeitos de rugosidade da parede de modo mais eficiente (modelos híbridos ou Lagrange-Euler com modelo de colisão) e obtenção de dados experimentais quanto ao detalhamento da fase particulada.

A utilização de CFD-DEM com submodelo para considerar efeitos de rugosidade nas paredes para o choque das partículas é uma alternativa interessante nesse caso, pois também tornaria mais simples a implementação das forças de coesão necessárias para considerar a aglomeração, característica esperada para materiais sólidos úmidos.

\section{CONCLUSÕES}

Os resultados das simulações EulerEuler indicaram que a alimentação na vertical apresentou melhor homogeneidade para a fase sólida. Como a configuração também promove menores perdas de carga e abrasão, ela se apresentou como a alternativa mais apropriada para aplicações em secagem e préaquecimento de material particulado. Este é o primeiro resultado que procura utilizar ferramentas na análise desta questão, que é ponto chave para a utilização de secadores flash em trecho ascendente ou em configuração circulante.

Porém, faltam dados experimentais obtidos nas condições simuladas e modelos Euler-Euler já apresentaram problemas para simulação de efeitos em curva, segundo 


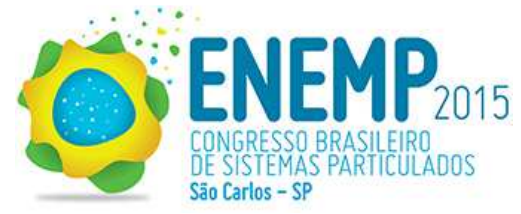

revisão da literatura. Assim, o trabalho aponta para a necessidade de obter dados experimentais quanto ao detalhamento do comportamento do material particulado no sistema e para o aprimoramento da modelagem da alimentação na horizontal seguida por curva.

\section{REFERÊNCIAS}

COSTA I A; CARMO M F; FREIRE J T. 2004. Analysis of regime transitions and flow instabilities in a vertical conveying of coarse particles using different solids feeding systems. The Canadian Journal Of Chemical Engineering, v.82, p.48-59.

DU B.; WARSITOW.; FAN L. S. 2004. ECT studies of the choking phenomenon in a gas-solid circulating fluidized bed. AIChE J. 50, 1386-1406.

GIDASPOW, D.; BEZBURUAH, R.; DING J. 1992. Hydrodynamics of circulating fluidized beds, kinetic theory approach. Fluidization VII, Proceedings of the $7^{\text {th }}$ Engineering foundation conference on fluidization; Potter , O.E.; Nicklin, D.J. (Eds.). Engineering foundation: New York; pp 75-82.

GRACE J R; TAGHIPOUR F. 2004. Verification and validation of CFD models and dynamic similarity for fluidized beds. Powder Technology, v.139, p.99-110.

GRBAVCIC Z B; GARIC-GRULOVIC R V; ARSENIJEVIC Z LJ. 2006. Prediction of the choking velocity in vertical pneumatic conveying of coarse particles. v.161, p. 1-9.

HIDAYAT, M.; RASMUSON, A. 2007. Heat and mass transfer in U-bend of a pneumatic conveying dryer. Chem. Eng. Res. \& Design, 85, 307-319.
LOPES C.S. 2011. Influência do alimentador de sólidos na fluidodinâmica do transporte pneumático. Tese de doutorado, programa de pós graduação em eng. química, UFSCar.

LOPES, C. S.; PÁDUA, T. F.; FERREIRA, M. C.; FREIRE, J. T. 2011. Influence of the entrance configuration on the performance of a non-mechanical solid feeding device for a pneumatic dryer. Drying Technol., 29, 1186-1194.

LUN, C. K. K.; SAVAGE, S. B.;JEFFREY,D. J.; CHEPURNIY N. 1984. Kinetics theories for granular flow: inelastic particles in couette flow and slightly inelastic particles in general flow field. J. Fluid Mech. 140, 223-256.

PÁDUA T.F.; LOPES C.S; FREIRE J.T. Estudo experimental sobre a alimentação de sólidos em dispositivos Venturi de orientação vertical para transportadores pneumáticos de material particulado. 2011. XXXV Congresso Brasileiro de Sistemas Particulados, 2011, Vassouras - RJ. Anais... Vassouras, UFRRJ. p.10.

PÁDUA T.F.; BÉTTEGA R.; FREIRE J.T. 2015. Gas-solid flow behavior in a pneumatic conveying system for drying appliations: coarse particles feeding with a venture device. Advances Chemical Engineering and Sciance, in press.

PIRKER S; KAHRIMANOVIC D; KLOSS C; POPOFF B; BRAUN M. 2010. Simulating coarse particle conveying by a set of Eulerian, Lagrangian and hybrid particle models. Powder Technology, v. 204, p. 203-213.

RAJAN, K. S.; DHASANDHAN, K.; SRIVASTAVA, S. N.; PITCHUMANI B. 2008. Studies on gas-solid heat transfer 
during pneumatic conveying. Int. J. Heat Mass Transfer, 51, 2801-2813.

RAJAN K S; SRIVASTAVA $S \quad \mathrm{~N}$; PITCHUMANI B; DHASANDHAN K. 2008. Experimental study of thermal effectiveness in pneumatic conveying heat exchanger. Applied Thermal Engineering, v. 28, p. 1932-1941.

RAJAN, K S; SRIVASTAVA, S N; PITCHUMANI, B; SURENDIRAN, V. 2010. Thermal conductance of pneumatic conveying preheater for air-gypsum and air-sand heat transfer. International Journal of Thermal Sciences, v.49, p.182-186.

RAJAN, K. S.; SRIVASTAVA, S. N.; PITCHUMANI, B.; MOHANTY, B. 2006. Simulation of gas-solid heat transfer during pneumatic conveying: Use of multiple gas inlets along the duct. Int. Comm. Heat Mass Transfer, 33, 1234-1242.

SOUSA, R. C.; ALMEIDA, A. R. F.; FERREIRA, M. C.; FREIRE, J. T. 2010. Analysis of Fluid Dynamics and Thermal Behavior Using a Vertical Conveyor with a Spouted Bed Feeder. Drying Technol. 28, 1277-1287.

VASHISTH S; GRACE J R. 2012. Simulation of Granular Transport of Geldart Type-A, -B, and -D Particles through a 90 degrees Elbow. Industrial \& Engineering Chemistry Research, v. 51, p. 2030-2047.

WEN, C. Y.; YU, Y. H. 1966. Mechanics of fluidization. Chem. Eng. Progress symp. Series. 162, 100-111.

\section{AGRADECIMENTOS}

Os autores agradecem à CAPES e ao $\mathrm{CNPq}$ pelo apoio financeiro. 